\title{
Electrical Properties of Nanocomposites
}

\author{
Mashal Alenazi \\ University of Bridgeport, Biomedical Engineering, USA
}

\begin{abstract}
A series of carbon nanofiber (CNF)/ polydimethylsiloxane (PDMS)-based nanocomposites was prepared using in situ polymerization technique by critical manipulation of factors, such as method of preparation and chemical modification of filler. Quantification of the degree of dispersion was done by introducing a dispersion degree parameter. Ceria-based composites are developed as potential electrolytes for intermediate solid oxide fuel cell applications and a better understanding of the ionic conduction mechanism serves this purpose. Ceria based composites were produced by several processing routes using a ceria-based ceramic host ( $\mathrm{Sm}$, Gddoped ceria) and various $\mathrm{Na}$ and Li carbonates. Simple ceria/carbonate two-phase nanocomposites were synthesized by the carbonate precipitation method followed by a thermal treatment.
\end{abstract}

Keywords: Electrical, Nanocomposites, CNF, Nano fillers.

\section{INTRODUCTION}

A problem of morphology of various materials with complex structure such as various nanocomposites structures becomes ever increasingly significantly in different areas. Specification of such structures requires topological and geometrical descriptors so we are able to characterise the connectivity and spatial configuration. In the last years, new advanced techniques have been developed in spatial statistics. Generally, it may be stated that today there is strive for use of multipoint statistic correlation functions for morphology analysis. The electric properties of composite or nanocomposite films come in on foreground of interest, especially near the percolation threshold. The parameter which influences the electric properties of composite structures is the metal volume fraction [1] contained in dielectric material. The metal particles are completely insulated from each other at low values of metal volume fraction, near the critical value of it they form a percolation structure, and the structure embodies the metallic behaviour with dielectric inclusions above this value. However, the percolation threshold can have a wide range, from a little as $15 \%$ metal all the way to $80 \%$. One can suppose ohmic or tunnelling conductivity in the structures. In the case of ohmic conductivity so called infinite cluster - objects connected to both electrodes applied to opposite sides of the structure - is analysed by the help of modified burning method, which takes into account spherical objects with real centre coordinates.

It is anticipated that much lower amount of nanofillers can be used to reinforce rubbers as compared to microscale fillers due to the higher specific surface area of the former. For example, incorporation of $10 \mathrm{wt} \%$ layered silicates in natural rubber resulted in significant increase in modulus $[3,4]$. Of late, emergence of nanofillers such as carbon nanotube (CNT) has evoked much interest in these materials as potential reinforcing filler in natural rubber However, the dispersion of CNT into rubbers continues to remain a challenge due to the high-aspect ratio of CNT and high viscosity of rubber. Methods to achieve improvement in the homogenous dispersion of CNT in the polymer matrix include use of dispersion aids or surface active agents and chemical functionalization of the CNT surface. Elastomers usually exhibit low electrical conductivity (less than 10-11 S/m). But, the addition of conductive nanofillers can improve their electrical conductivity up to $10-2 \mathrm{~S} / \mathrm{m}$. The unique electrical conductive behavior is attributed to the formation of percolation pathways of the conductive fillers in the matrices, which leads to a sudden increase in the conductivity at a critical filler concentration, commonly referred to as the percolation threshold An easy way to comply with the conference paper formatting requirements is to use this document as a template and simply type your text into it.

The surfactant was incorporated on the surface of the CNT in two steps. The first step was to incorporate oxygen containing functional groups $(-\mathrm{OH}$ and $-\mathrm{COOH})$ on the CNT to enhance the reactivity. Each $1 \mathrm{~g}$ of the CNT was treated with $10 \mathrm{~mL}$ of concentrated nitric acid at a temperature of $120 \mathrm{C}$ for $48 \mathrm{~h}$ under continuous stirring on a magnetic stirrer. It was washed with deionized water to remove excess nitric acid. In the second step, the acid treated CNT was reacted with the surfactant, 1octadecanol. This involves heating of 1-octadecanol to its melting point in a reaction flask and was maintained slightly below its boiling point, while CNT was added into the reaction flask containing the molten surfactant in the CNT: surfactant ratio of 1:10 parts by weight. The mixture was stirred for a few minutes and a few drops of sulfuric acid were added to initiate the reaction. The reaction was allowed to continue for $2 \mathrm{~h}$ beyond which the resulting CNT was washed with toluene several times to remove any unreacted 1-octadecanol, followed by washing with deionized water to remove any acid traces (Scheme 1). The resulting CNT is referred to as $\mathrm{C} 18-\mathrm{CNT}$ in this article. Extensive literature survey reveals that no work has been done till date on CNF/PDMS nanocomposites. The probable reason for this is the huge surface energy difference of PDMS and CNF. PDMS is a semiorganic polymer with very low surface energy $(* 19.6 \mathrm{~mJ} / \mathrm{m})$ whereas CNF has an appreciably high surface energy of $145-165 \mathrm{~mJ} / \mathrm{m} 2$. This restricts homogeneous dispersion 
of nanofibers in the polymer matrix. Thus, preparation of activity toward electro-oxidation of organic compounds $\mathrm{CNF} / \mathrm{PDMS}$ nanocomposites is itself challenging and containing $\mathrm{OH}$ and $\mathrm{NH}$ functional groups. Hydrazine as an novel. Moreover, the method of in situ preparation of important example of NH2containing compounds is a 2 nanocomposite through anionic ring opening strong reducing agent widely used in industrial factories polymerization is completely new. In this article, it is and agricultural applications [4]. Of note, hydrazine may shown for the first time how the in situ preparation of cause some carcinogenic and hepatotoxic effects. It may nanocomposite affects the extent of dispersion in cause liver and kidney damages, also irreversible comparison with the conventional ex situ prepared deterioration of nervous system when it absorbs through nanocomposites [2].

\section{APPROACHES TO GNPS}

GNPs can be synthesized through chemical vapour deposition and arc discharging methods, among others (just as other graphitic carbon nanofillers, owing to the plentiful graphite in nature), two fabrication approaches are most preferred: mechanical milling and graphite intercalation chemistry approaches. Mechanical milling [8] is a top-down process by breaking up bulk graphite, especially breaking the Van de Waals force. The resulting GNPs have the disadvantages of large particle size and a broad particle size distribution. These problems could be readily improved by the graphite-intercalation approach, which starts from the intercalation of graphite to obtain graphite intercalate compound (GIC). The basis of intercalation is to "place" some chemicals within the natural graphite layers. These chemicals are able to react and yield either a large volume of gas or enormous heat to force the separation of adjacent grapheme layers. Acid intercalation is the most mature and commonly used technique for this purpose. It is usually done by immersing natural graphite in concentrated/fumed sulfuric acid $(\mathrm{H} 2 \mathrm{SO})$ solution containing various oxidizers such as concentrated nitric acid (HNO43), $\mathrm{KMnO} 4, \mathrm{H} 2 \mathrm{O}$, etc. The acid intercalation can also be carried out by vapour phase reaction and electrochemical methods [5].

\section{III.FABRICATION OF POLYMER NANOCOMPOSITE}

Currently conducting polymers are important materials play important roles in a wide variety of applications including energy storage, microelectronics, electro chromic displays, electro catalysis and chemical sensors [1-4]. An important class of conducting polymers is conducting metallopolymers, containing various metal centers from main groups or transition metals. The metal centers can be located either in the polymer main chain or in the side group structure [5]. Recently, modification of electrodes with conducting polymers, especially metallopolymers, has attracted enormous attentions. Among the various procedures for modification of electrodes, electrochemical polymerization is an effective, controllable and facile strategy to prepare metallopolymers coated electrodes.

Electrochemical modification usually proceeds by oxidation (or reduction) of an appropriate metal complex monomer to form a polymeric film. Electrochemistry of nickel and nickel-based electrodes in the alkaline media is an important area for researchers. The electrode modified with thin film of polymers of nickel macro cycle (salen, porphyrins, phthalocyanines, etc.). shows high catalytic skin. Accordingly, determination of hydrazine in environmental and medical samples isof high importance. Thus, several methods have been employed for this purpose; including potentiometry, fluorimetry and spectrophotometry. However, the over potential of hydrazine electro-oxidation is usually high at conventional electrodes, thusa wide variety of modified electrodes have been developed to decrease this over potential and enhance the electron transfer rate. Among them, nanoparticles modified surfaces have attracted widespread interest in recent years due to the substantial enhancement in electron transfer rate and catalytic properties,

\section{RESULTS AND DISCUSSION}

In order to find the optimum potential for fabrication of $\mathrm{Ni}-\mathrm{NPs}$, cyclic voltammogram of GC electrode in nickel nitrate solution was recorded (Fig. 1); and four potentials were chosen accordingly. As shown in Fig. 1, the reduction of nickel ions and deposition of NiNP stake places at potentials more negative than $-0.8 \mathrm{~V}$. However, at potentials more negative than $-1.3 \mathrm{~V}$, it was seen that electro deposition may be prevented because of the formation of large $\mathrm{H}$ bubbles. Hence, the potential of -1.3 $\mathrm{V}$ was chosen as electro deposition potential in potential step procedure. A plot of peak current against deposition time for Ni-NPs deposition on the GC electrodes figure 1.

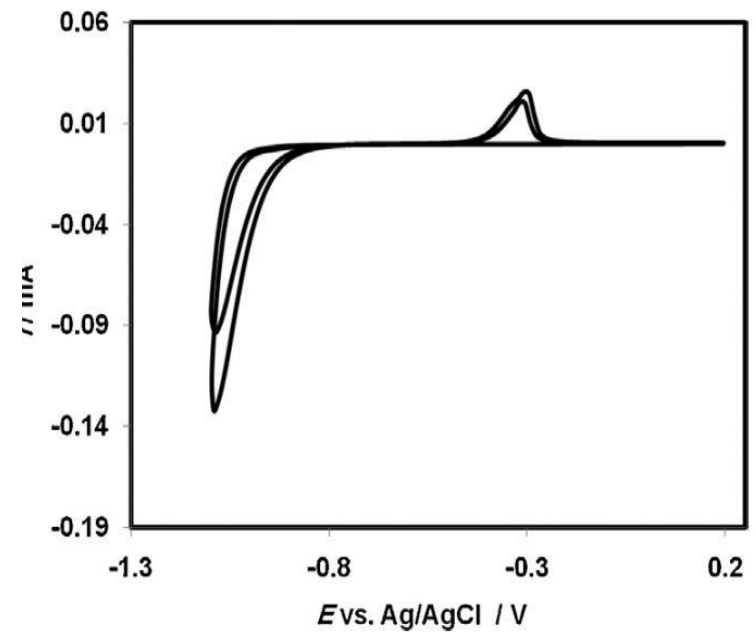

Figure 1. Cyclic voltammograms

Though the elastomeric PDMS matrix is insulating in nature, proper dispersion of the $\mathrm{CNF}$ in the matrix results in significant improvement in electrical conductivity. However, good dispersion is not just the only criteria for enhanced electrical properties. Good dispersion of nanofibers. But the resultant hybrid material shows poor conductivity almost similar to that of the unfilled 
elastomer. On the other hand, good dispersion and the nanocomposite shows good conductivity. The difference lies in the fact that in the latter one a conducting network is formed by the nanofibers which is absent in the former.

Thus, formation of a conducting network facilitates improvement in electrical and dielectric properties of the nanocomposites. Both CNT and C18-CNT were characterized using Fourier transform infrared spectroscopy (Perkin Elmer 16F PC FTIR) instrument. FTIR samples were prepared by grinding dried functionalized CNT together with potassium bromide $(\mathrm{KBr})$ to make a pellet. The DC volume resistivity of the composite materials having wide range of resistivity was measured using instruments Agilent 4339B (Highresistance meter attached with agilent $16008 \mathrm{~B}$ resistivity cell) for measurement range covering from 1016 to 106 ohm and GOM-802 (GW Instek DC milli Ohm Meter) for low resistance measurement. GOM-802 is attached with a homemade electrode, covering range from 106 to $10-30$ phenyl-paraphenylenediamine (antioxidant), TMQ 2,2,4trimethyl-1,2-dihydroquinoline (antioxidant), TMTD tetramethyl thiuram disulfide (accelerator), MBT 2mercaptobenzothiazole (accelerator) ohm. Tensile tests were carried out using Zwick tensile testing machine with specimen grip of $50 \mathrm{~mm}$ and testing speed of $500 \mathrm{~mm} / \mathrm{min}$. NR compounds containing 1, 5, and $10 \%$ of CNT and C18-CNT were molded on a hot press under a pressure of $350 \mathrm{kPa}$ at a temperature of $150 \mathrm{C}$ for $15 \mathrm{~min}$ in order to make flat sheets of $2 \mathrm{~mm}$ thickness. Five specimens each of $35 \mathrm{~mm}$ in length and $2 \mathrm{~mm}$ in thickness were prepared from each batch and tested according to ASTM D412. Philips CM200 TEM was used with a field emission gun (FEG), which provides very high resolution for the TEM measurements under high vacuum.

The samples were prepared using a Leica FC6 Cryoultramicrotome. A small piece of the sample (middle) was cut and mounted in cellulose and the sample was cut by diamond knife. The slices were cut into $60 \mathrm{~nm}$ size.

\section{CONCLUSION}

I have seen that the structures of the current paths - fuzzy clusters - strongly depend on the degree of arrangement of the objects in the structure. The fuzzy clusters become more regular with increasing degree of arrangement of the objects. The same dependence can be found in the potential distributions of objects. The next consequence of it is increasing total electric current and the number of current paths in the structure. The differences between currents flowing in individual current paths become smaller. A two-phase thermally stable ceria/carbonate composite has been prepared by precipitation followed by wet mixing which ensures an atomic level homogeneity. No ceria doping and no carbonate mixture were used for the preparation of the composite. The decomposition sequence of the precursor, the stability and the formation process of the composite were studied by TG-DTA, IR, UV and XRD analyses.

\section{REFERENCES}

[1] Bai, J. B., \& Allaoui, A. (2003). Effect of the length and the aggregate size of MWNTs on the improvement efficiency of the mechanical and electrical properties of nanocompositesexperimental investigation. Composites Part A: applied science and manufacturing, 34(8), 689-694.

[2] Sandler, J., Shaffer, M. S. P., Prasse, T., Bauhofer, W., Schulte, K., \& Windle, A. H. (1999). Development of a dispersion process for carbon nanotubes in an epoxy matrix and the resulting electrical properties. Polymer, 40(21), 5967-5971.

[3] Geng, Y., Liu, M. Y., Li, J., Shi, X. M., \& Kim, J. K. (2008). Effects of surfactant treatment on mechanical and electrical properties of CNT/epoxy nanocomposites. Composites Part A: Applied Science and Manufacturing, 39(12), 1876-1883.

[4] Allaoui, A., Bai, S., Cheng, H. M., \& Bai, J. B. (2002). Mechanical and electrical properties of a MWNT/epoxy composite. Composites Science and Technology, 62(15), 1993-1998.

[5] Yoon, J. T., Lee, S. C., \& Jeong, Y. G. (2010). Effects of grafted chain length on mechanical and electrical properties of nanocomposites containing polylactide-grafted carbon nanotubes. Composites Science and Technology, 70(5), 776-782. 